\section{Primera caracterización molecular de Leishmania infantum en pacientes con leishmaniosis visceral de la Provincia de Misiones, Argentina}

\author{
Ricardo G. Díaz¹, Karina A. Salvatierra1, Gustavo A Silva¹, Enrique J. Deschutter ${ }^{1}$,
} Fernando J. Bornay-Llinares ${ }^{2,}$ Lucrecia Acosta $^{2,3}$

${ }^{1}$ Cátedra de Parasitología, Facultad de Ciencias Exactas, Químicas y Naturales, Universidad Nacional de Misiones, Posadas, Misiones, Argentina

${ }^{2}$ Área de Parasitología, Departamento de Agroquímica y Medio Ambiente, Universidad Miguel Hernández de Elche, San Juan, Alicante, España

${ }^{3}$ Unidad de Biología Molecular, Sanatorio Fontilles, Alicante, España

Introducción. La leishmaniosis cutánea por Leishmania braziliensis ha sido tradicionalmente endémica en Argentina y se han sido descritos casos de compromiso visceral después de una leishmaniosis cutánea inicial. La leishmaniosis visceral emergió en Argentina en el año 2006 en la ciudad de Posadas, provincia de Misiones, afectando tanto a humanos como a perros.

Objetivo. Identificar el agente etiológico a nivel de especie de los pacientes diagnosticados con leishmaniosis visceral en Misiones y describir sus características clínico-

epidemiológicas.

Materiales y métodos. Se estudió una serie de 24 pacientes con diagnóstico confirmado de leishmaniosis visceral en la provincia de Misiones en el período 2009 al 2016. Para la identificación de Leishmania spp., los pacientes fueron sometidos a estudios diagnósticos indirectos (serológicos) y directos (microscopía, detección de ADN y secuenciación). También, se estudiaron variables como edad, sexo, lugar de residencia, y signos y síntomas clínicos indicativos de leishmaniosis visceral.

Resultados. De los 24 pacientes estudiados, 18 (75\%) eran hombres y 6 (25\%) eran menores de cuatro años. La manifestación clínica más frecuente fue el síndrome febril prolongado en 21 (87,5\%) de los pacientes, seguido de esplenomegalia en 17 (70,8\%). Se identificó la especie Leishmania infantum en todos los pacientes estudiados.

Conclusión. Este hallazgo constituye la primera identificación de la especie $L$. infantum en pacientes autóctonos de la provincia de Misiones. El estudio evidenció la importancia de la PCR para el manejo epidemiológico de la leishmaniosis visceral en Argentina.

Palabras clave: Leishmania infantum; reacción en cadena de la polimerasa; leishmaniasis visceral; Argentina.

Lucrecia Acosta, Área de Parasitología, Universidad Miguel Hernández de Elche, Edificio Muhammad AlShafra, Campus de Sant Joan d'Alacant s/n, 03550

Sant Joan, Alicante, España

Teléfono: (0034) 965919350

lacosta@umh.es

Contribución de los autores:

Enrique J. Deschutter, Fernando J. Bornay-Llinares, Gustavo A Silva: conceptualización y diseño del estudio

Gustavo A Silva: estudios de laboratorio

Karina A. Salvatierra: caracterización molecular de las especies

Ricardo G. Díaz y Lucrecia Acosta participaron en todas las etapas del estudio.

Todos los autores participaron en la redacción y la corrección del manuscrito.

Financiación:

Este estudio se llevó a cabo con fondos para los proyectos de investigación incentivados y otorgados por la Facultad de Ciencias Exactas, Químicas y Naturales de la Universidad Nacional de Misiones, Resolución CD325-13, en el marco del proyecto "Optimización del diagnóstico de leishmaniasis visceral humana y canina en Misiones", así como por el Sanatorio Fontilles y el programa de cooperación para el desarrollo de la Universidad Miguel Hernández de Elche.

Conflicto de intereses:

Los autores declaran que no existen conflictos de intereses.
First molecular characterization of Leishmania infantum species in patients infected with visceral leishmaniasis in Misiones province, Argentina

Introduction: Cutaneous leishmaniasis caused by L. braziliensis has been historically endemic in Argentina and several cases of visceral leishmaniasis following initial cutaneous leishmaniasis have been reported. Visceral leishmaniasis started to appear in Argentina in 2006 in the city of Posadas, Misiones province, affecting both humans and dogs. Objective: To identify the etiologic agent to species level in patients with visceral leishmaniasis diagnosis in Misiones province and describe its clinical and epidemiological characteristics.

Materials and methods: A cohort of 24 patients from Misiones province was studied from 2009 to 2016, all with a confirmed diagnosis of visceral leishmaniasis. To identify the Leishmania species involved, patient samples were analyzed by microscopy, serologic studies, DNA detection, and sequencing. Variables such as age, sex, place of residence, clinical signs and symptoms consistent with visceral leishmaniasis were also recorded. Results: $75 \%$ (18/24) of the patients studied were males and $25 \%(6 / 24)$ were younger than 4 years. The most frequent symptom was a prolonged fever in $87.5 \%$ of the patients $(21 / 24)$, followed by splenomegaly in $70.8 \%(17 / 24)$. Leishmania infantum was the only parasite species identified in all patients.

Conclusion: This finding constitutes the first molecular identification of the Leishmania infantum species in autochthonous patients of Misiones province, Argentina. This study highlights the importance of PCR for species identification in epidemiological studies of visceral leishmaniosis in Argentina.

Keywords: Leishmania infantum, polymerase chain reaction, leishmaniasis, visceral; Argentina. 
Las leishmaniosis engloban un conjunto de enfermedades parasitarias de transmisión vectorial que afectan tanto al ser humano como a otros vertebrados. Estas parasitosis son causadas por diferentes especies de protozoos intracelulares del género Leishmania, que son transmitidos por picaduras de las hembras hematófagas de la subfamilia Phlebotominae (1).

Las leishmaniosis son endémicas en 98 países de cinco continentes y se diagnostican anualmente 1,3 millones de nuevos casos, de los cuales 300.000 son de leishmaniosis visceral. Más de 310 millones de personas viven en zonas de riesgo de contraer leishmaniasis visceral y de $20.000 \mathrm{a}$ 50.000 personas mueren anualmente (2).

Esta leishmaniasis es mundialmente reconocida como una de las enfermedades infecciosas más importantes y afecta principalmente a la población pobre que vive en áreas rurales y suburbanas; es endémica en la mayoría de las regiones tropicales y subtropicales tanto del Nuevo como del Viejo Mundo (3).

En un estudio realizado en la ciudad de Posadas, se concluyó que la mala calidad de la vivienda y el hacinamiento (pobreza estructural) se asociaban positivamente con el incremento del riesgo de contraer leishmaniasis visceral humana (4). En los focos registrados en la provincia de Misiones es característica la urbanización no planificada que intercala áreas verdes y de uso doméstico, donde la cantidad de material orgánico es abundante, lo cual favorece la colonización y la persistencia del vector Lutzomyia longipalpis. Estas características ambientales y socio-geográficas se asocian, por lo general, con deficientes servicios sanitarios y dificultades de acceso al sistema de salud, lo que, a su vez, representa un factor adverso para el diagnóstico temprano de leishmaniasis visceral (5).

En el continente americano, la especie Leishmania infantum es el principal agente etiológico de la denominada leishmaniosis visceral zoonótica (1). Se trata de una enfermedad que puede afectar tanto al ser humano como a otros mamíferos -perros domésticos, zorros, lobos, linces, coatíes, ratas, comadrejas y osos hormigueros, entre otros-. Aunque tradicionalmente se ha considerado como una enfermedad rural, hoy en día se encuentra bien establecida en zonas urbanas $(1,3,6)$, en las cuales el perro es el principal reservorio $(1,7)$.

La especie $L$. infantum infecta, principalmente, macrófagos de órganos hematopoyéticos -médula ósea, bazo e hígado-, causando graves disfunciones orgánicas.

En Posadas (Misiones, Argentina), se detectó el primer caso autóctono de leishmaniasis visceral humana en el 2006 (8). Hasta hoy, se han detectado más de 100 casos con una mortalidad del $10 \%$, tal como se ha descrito en otras regiones del mundo $(9,10)$.

La leishmaniasis visceral en Argentina se encuentra en expansión, así como sus vectores (11-13), y se han detectado casos humanos en otras cuatro provincias: Salta, Entre Ríos, Santa Fe y Santiago del Estero (12,14-17).

El presente estudio tuvo como objetivos describir sus características clínicas y epidemiológicas, e identificar, mediante reacción en cadena de la polimerasa (PCR), la especie o especies del género Leishmania involucradas en una serie de pacientes con leishmaniasis visceral confirmados por el sistema nacional de vigilancia en salud de Argentina en el período 2009 a 2016 en la provincia de Misiones. Asimismo, se evaluó la utilidad de esta técnica para el diagnóstico y los estudios epidemiológicos. 


\section{Materiales y métodos}

\section{Área de estudio}

La provincia de Misiones posee un clima típicamente subtropical: caluroso, con abundantes lluvias y alta humedad relativa. Comprende una superficie de $29.801 \mathrm{~km}^{2}$ y limita al norte y al este con Brasil, y al oeste con Paraguay. Según el censo del Instituto Nacional de Estadísticas y Censos (INDEC), en el 2010 Misiones contaba con 1'101.593 habitantes, de los cuales el 49,68\% eran hombres (18).

\section{Pacientes}

Se seleccionaron los pacientes de la provincia de Misiones que concurrieron a consulta clínica al Hospital Escuela de Agudos "Ramón Madariaga" entre el 2009 y 2016, resultaron ser casos confirmados de leishmaniasis visceral y en quienes se logró amplificar mediante PCR el ADN correspondiente al género Leishmania.

Finalmente, fueron objeto de estudio 24 pacientes. Estos cumplieron los siguientes criterios de inclusión:

i. residir en la provincia de Misiones, concurrir a consulta clínica y resultar caso sospechoso de leishmaniasis visceral;

ii. dar el consentimiento para usar el material clínico en los estudios de caracterización del agente etiológico, y

iii. tener, al menos, un resultado positivo en alguna de las siguientes técnicas: inmunocromatografía, examen directo por microscopía o estudios moleculares (PCR).

No se incluyó el total de pacientes del período de estudio por no cumplir todos ellos con los criterios de inclusión.

\section{Toma de muestra y recolección de datos}

Las fichas clínico-epidemiológicas en las que el profesional médico asentó los datos pertinentes del paciente, incluían la siguiente información: fecha de toma de la muestra, edad, sexo y localidad de residencia. El laboratorio no recibe datos que identifiquen al paciente.

Los síntomas y signos clínicos registrados en el estudio fueron la presencia o ausencia de fiebre prolongada, pérdida de peso, hepatomegalia, esplenomegalia, palidez, ictericia, edema y ganglios linfáticos.

Para los estudios de laboratorio se tomaron dos tipos de muestras: una de médula ósea tomada mediante punción-aspiración de aproximadamente $1,5 \mathrm{ml}$ con $50 \mu \mathrm{l}$ de $\operatorname{EDTA}(0,342 \mathrm{M})$, y $3 \mathrm{ml}$ de sangre periférica mediante punción venosa practicada por el personal del laboratorio de la institución a la cual acudió el paciente. Los tubos con sangre se centrifugaron por 5 minutos a $3.000 \mathrm{rpm}$ para recuperar el suero.

\section{Técnicas diagnósticas}

Técnicas diagnósticas indirectas (serológicas)

Test inmunocromatográfico rápido basado en el antígeno recombinante rK39 (ICT-rK39). Se analizó el suero de los pacientes seleccionados con tiras 
reactivas Kalazar Detect Rapid Test ${ }^{\mathrm{TM}}$ (InBIOS International Inc., Seattle, WA) para humanos. La prueba inmunocromatográfica se practicó siguiendo las instrucciones del fabricante.

\section{Técnicas diagnósticas directas}

Microscopía óptica. Los extendidos de médula ósea tomada mediante punción-aspiración se tiñeron con Giemsa y se examinaron al microscopio óptico en busca de amastigotes de Leishmania spp.

\section{Técnicas moleculares}

i. Extracción de ácidos nucleicos. Todas las muestras de médula ósea se procesaron mediante la columna NucleoSpin Tissue de Macherey$\mathrm{Nagel}^{\mathrm{TM}}$ (Bethlehem, USA), siguiendo las instrucciones del fabricante. Como control negativo de extracción, se usó una muestra negativa de médula ósea suministrada por el Hospital Escuela de Agudos "Dr. Ramón Madariaga".

ii. Detección de ácidos nucleicos. Se amplificó la región del espaciador interno de la transcripción 1 (ITS-1) mediante PCR anidada. El protocolo utilizado para la primera reacción se basó en el que desarrollaron Schönian, et al. (19), incluyendo las modificaciones de Cruz, et al., para la segunda reacción (20). Con el fin de determinar el buen funcionamiento de los reactivos y su manipulación, se usaron un control negativo (agua bidestilada estéril) y un control positivo (ADN obtenido del aislamiento de cultivo suministrado por el Instituto Nacional de Parasitología "Dr. Mario Fatala Chabén"), en cada una de las reacciones de PCR. La visualización de los productos se realizó en un gel de agarosa al 1,5\% teñido con bromuro de etidio.

iii. Caracterización. Este procedimiento se realizó mediante la secuenciación directa de los productos de PCR con los cebadores específicos de la segunda reacción (20), y usando el estuche comercial para secuenciación de ciclos de terminación Big-Dye Terminator Cycle Sequencing Ready Reaction KitTM, versión 3.1, y el secuenciador de ADN automático ABI Prism 377 ${ }^{\text {TM }}$ (Applied Biosystems, Foster City, CA). Las secuencias obtenidas se analizaron y se editaron con el programa analizador de secuencias Lasergene ${ }^{\mathrm{TM}}$ (DNASTAR, Madison, WI). Posteriormente, las secuencias obtenidas se compararon en la base de datos GenBank para verificar la coincidencia con alguna especie descrita.

\section{Aspectos éticos}

Todos los procedimientos que se llevaron a cabo fueron revisados y aprobados por el Comité de Bioética del Hospital Público Provincial de Pediatría "Dr. Fernando Barreyro" de la ciudad de Posadas. Los protocolos de la toma de muestra y de su manejo se realizaron según las directrices del Hospital Escuela de Agudos "Dr. Ramón Madariaga".

Todos los participantes del estudio dieron su consentimiento por escrito, según el modelo de consentimiento informado utilizado por este hospital para los estudios de leishmaniasis visceral. Dicho consentimiento avaló la realización de técnicas diagnósticas para la identificación de la especie de Leishmania. 


\section{Resultados}

Se estudiaron 24 pacientes con diagnóstico de leishmaniasis visceral confirmado mediante observación microscópica directa, test inmunocromatográfico rápido o PCR.

Los pacientes residían mayoritariamente en Posadas (8/24; $33 \%)$. Las demás localidades de residencia fueron: Oberá, con 6 (25\%); San Ignacio, con 5 (20\%); Candelaria, con 3 (12,5\%); Apóstoles, con 1 (4\%), y Alem, con 1 (4\%).

De los 24 pacientes estudiados, 18 (75\%) eran hombres y 6 (25\%) eran menores de cuatro años. En el cuadro 1 se presentan los resultados de laboratorio y la distribución por sexo y edad de los pacientes.

El paciente asintomático fue un neonato, a cuya madre se le diagnosticó leishmaniasis visceral durante el embarazo. Ningún paciente presentó todos los síntomas, aunque dos de ellos presentaron seis síntomas simultáneos.

Cuadro 1. Resultados de laboratorio y características epidemiológicas de los pacientes relacionadas con las variables de edad y sexo

\begin{tabular}{lllrc}
\hline ICT & Frotis & PCR & $\begin{array}{r}\text { Edad } \\
\text { (años) }\end{array}$ & Sexo \\
\hline Positivo & Negativo & Positivo & 71 & $\mathrm{M}$ \\
Positivo & Positivo & Positivo & 41 & $\mathrm{M}$ \\
Positivo & Positivo & Sin dato & 2 & $\mathrm{M}$ \\
Positivo & Positivo & Positivo & 46 & $\mathrm{M}$ \\
Positivo & Negativo & Sin dato & 75 & $\mathrm{~F}$ \\
Positivo & Negativo & Sin dato & 44 & $\mathrm{M}$ \\
Positivo & Negativo & Positivo & 58 & $\mathrm{M}$ \\
Positivo & Positivo & Positivo & 1 & $\mathrm{M}$ \\
Positivo & Positivo & Positivo & 3 & $\mathrm{~F}$ \\
Positivo & Positivo & Positivo & 20 & $\mathrm{~F}$ \\
Positivo & Positivo & Positivo & 42 & $\mathrm{M}$ \\
Positivo & Positivo & Sin dato & 13 & $\mathrm{~F}$ \\
Negativo & Negativo & Positivo & 78 & $\mathrm{M}$ \\
Positivo & Positivo & Positivo & 2 & $\mathrm{M}$ \\
Positivo & Negativo & Positivo & 37 & $\mathrm{M}$ \\
Positivo & Negativo & Positivo & 39 & $\mathrm{M}$ \\
Positivo & Positivo & Sin dato & 0 & $\mathrm{M}$ \\
Positivo & Positivo & Positivo & 1 & $\mathrm{~F}$ \\
Positivo & Positivo & Positivo & 53 & $\mathrm{M}$ \\
Positivo & Positivo & Positivo & 11 & $\mathrm{M}$ \\
Positivo & Positivo & Positivo & 34 & $\mathrm{M}$ \\
Positivo & Positivo & Positivo & 36 & $\mathrm{M}$ \\
Negativo & Positivo & Positivo & 24 & $\mathrm{~F}$ \\
Positivo & Negativo & Positivo & 3 & $\mathrm{M}$ \\
\hline
\end{tabular}

ICT: test inmunocromatográfico rápido; PCR: reacción en cadena de la polimerasa indicativa de Leishmania infantum; M: masculino; F: femenino

En el cuadro 2 se presentan los signos y síntomas clínicos. La manifestación clínica más frecuente resultó ser el síndrome febril prolongado en 21 (87,5\%) pacientes, seguido de esplenomegalia en 17 (70,8 \%). Ningún paciente de esta serie presentó ascitis.

De los 24 pacientes estudiados, 19 (79,2\%) resultaron positivos por PCR anidada para el género Leishmania y $16(66,6 \%)$, mediante el frotis. El test inmunocromatográfico rápido resultó positivo en 22 (91,6\%) de los casos. 
Cuadro 2. Manifestaciones clínicas de los pacientes $(n=24)$

\begin{tabular}{lrr}
\hline \multicolumn{1}{c}{ Síntomas } & $\mathbf{n}$ & $(\%)$ \\
\hline Síndrome febril prolongado & 21 & $(87,5)$ \\
Pérdida de peso & 10 & $(41,7)$ \\
Hepatomegalia & 14 & $(58,3)$ \\
Esplenomegalia & 17 & $(70,8)$ \\
Palidez & 8 & $(33,3)$ \\
Ictericia & 2 & $(8,3)$ \\
Edema & 1 & $(4,16)$ \\
Ganglios linfáticos & 2 & $(8,3)$ \\
Ascitis & 0 & $(0,0)$ \\
\hline Fuente: Ministerio de Salud Pública, Provincia de Misiones
\end{tabular}

Todas las muestras que resultaron positivas por PCR anidada y confirmadas como Leishmania spp., se sometieron a secuenciación. En total, 19 muestras de leishmaniasis visceral fueron sometidas a secuenciación y todas las secuencias obtenidas presentaron una identidad del $100 \%$ con la especie L. infantum.

\section{Discusión}

El presente estudio constituye el primer trabajo de caracterización molecular de $L$. infantum de los casos de leishmaniasis visceral acontecidos en los últimos años en la provincia de Misiones.

Hasta el año 2006, en que se reportó el primer caso de leishmaniasis visceral autóctono de la Argentina, se habían descrito 17 casos de leishmaniasis visceral dentro del área endémica de leishmaniasis cutánea por $L$. braziliensis y, finalmente, se consideró que todos los casos autóctonos de la forma visceral se habían iniciado como una leishmaniasis cutánea producida por $L$. braziliensis (21). Los casos se describieron como autóctonos de las provincias del norte argentino (ninguno de ellos en Misiones) y cuatro de los casos se consideraron como importados de otros países. Solo un caso fue causado por $L$. infantum en la provincia de Mendoza y fue de origen desconocido (22).

Este hallazgo supone un cambio del cuadro epidemiológico de la leishmaniosis en Argentina. A pesar de que la provincia de Misiones es endémica para la leishmaniasis cutánea desde 1998 (23), y la especie $L$. braziliensis puede ser ocasionalmente viscerotrópica en el país, la única especie encontrada en estos pacientes con leishmaniasis visceral en Misiones fue $L$. infantum.

Estos resultados ponen de manifiesto la necesidad de la caracterización molecular del agente causal de la leishmaniasis visceral para un correcto manejo de los pacientes y un adecuado conocimiento de la epidemiología de la leishmaniosis en el país.

En Argentina la técnica de referencia para el diagnóstico de la leishmaniasis visceral es la demostración de los parásitos en el material de la punción esplénica o de médula ósea, la que brinda un diagnóstico de certeza $(1,24)$. Sin embargo, debido a la baja sensibilidad de la observación microscópica, el Ministerio de Salud de la Nación (Argentina) recomienda el uso de técnicas complementarias para su diagnóstico (24). En el presente estudio, el 66,6\% de los casos fue positivo a partir del extendido de muestras de médula ósea obtenida mediante punción aspiración, tal como se esperaba de acuerdo con lo observado por otros autores (25-27). 
En este sentido, se recomienda el test inmunocromatográfico rápido basado en el antígeno recombinante rK39 (ICT-rK39) como herramienta diagnóstica para la leishmaniasis visceral, considerando sus elevadas sensibilidad y especificidad. Si bien la detección de anticuerpos no implica la presencia de infección activa $(24,25)$, esta es considerada -junto con el cuadro clínico- la prueba de referencia para confirmar los casos humanos de leishmaniasis visceral en Argentina. El test inmunocromatográfico rápido ICT-rK39 resultó positivo en el 91,6\% de los pacientes estudiados, lo cual coincide con lo señalado por diversos autores (del 80 a 90 \%) (25).

Actualmente, los estudios moleculares han demostrado ser útiles para su diagnóstico y su caracterización rápida, temprana, y de alta sensibilidad y especificidad. Esta técnica es recomendada también por el Ministerio de Salud de la Nación para el diagnóstico de leishmaniosis visceral $(19,20,24,26,28)$. En el cual la técnica de PCR anidada podría ser una herramienta eficaz para caracterizar la especie causante. Por lo tanto, en el diagnóstico rutinario, la inclusión de la amplificación de los ácidos nucleicos seguida de la caracterización, podrían ser de utilidad para detectar esta infección en la provincia.

En el presente estudio los resultados obtenidos de la PCR anidada se asemejan a los obtenidos por otros autores (85-95\%), y se observó un número similar de posibles falsos negativos debidos a razones no esclarecidas, entre las que estarían la toma o la conservación de la muestra (28-31). La región diana empleada en este estudio (ITS-1) es lo suficientemente conservada para hacer el diagnóstico molecular y, además, presenta suficiente polimorfismo para permitir la tipificación a nivel de especie $(19,20,28-30)$.

El patrón epidemiológico en relación con los parámetros estudiados es diferente a lo observado en otras áreas. Según la Organización Mundial de la Salud (OMS) y la Dirección Nacional de Epidemiología y Análisis de Situación de Salud argentina, el $90 \%$ de los casos de leishmaniasis visceral ocurre en niños menores de 10 años $(1,24)$. Sin embargo, en este estudio no se encontró un patrón definido en cuanto a la edad de los pacientes, si bien es cierto que el $25 \%$ de ellos era menor de cuatro años.

Por otro lado, en los pacientes de Misiones se encontró un mayor porcentaje de hombres infectados, como se ha observado recientemente en otros estudios en Brasil $(32,33)$. La OMS puntualiza que en el pasado las leishmaniosis en las Américas habían sido históricamente enfermedades ocupacionales. Aunque los factores de riesgo ocupacionales siguen teniendo importancia, la presencia del vector en el ambiente peridoméstico ha llevado a un aumento rápido del número de casos de exposición ocupacional (1).

Los resultados obtenidos en el presente estudio sugieren que en esta área geográfica los factores ocupacionales podrían jugar un papel importante en la transmisión de la enfermedad, lo que se manifiesta con el predominio de casos en hombres adultos.

El movimiento migratorio en puntos como Posadas o Puerto Iguazú podría haber favorecido la aparición de la leishmaniasis visceral en la provincia, ya que en los países vecinos, Paraguay y Brasil, esta enfermedad se encuentra bien establecida; además, la presencia del vector aumenta la posibilidad de transmisión de esta enfermedad entre canes y humanos $(34,35)$. 
Las manifestaciones clínicas más frecuentes en los pacientes con leishmaniasis visceral fueron el síndrome febril prolongado y la esplenomegalia; esto concuerda con lo señalado por la OMS (1) y con los registros del sistema de vigilancia de los reportes oficiales del Ministerio de Salud de Misiones para los 116 pacientes notificados (1).

El único paciente asintomático fue un neonato incluido en el estudio, a cuya madre se le diagnosticó leishmaniasis visceral durante el embarazo. Existen evidencias de transmisión vertical de la leishmaniasis visceral, aunque resulta un hecho poco frecuente (36). El neonato de este estudio resultó positivo en las pruebas serológicas y en el frotis de la muestra de la médula ósea, aunque los resultados de las pruebas moleculares fueron negativos (36). Los resultados serológicos positivos en estos neonatos podrían resultar del paso transplacentario de anticuerpos maternos $(1,36,37)$. A pesar de no presentar síntomas, el paciente fue tratado con anfotericina B liposómica y fue dado de alta al completar el tratamiento.

En conclusión, en este estudio se presenta la primera identificación del agente etiológico de la leishmaniasis visceral en Misiones, Argentina, la cual confirmó la presencia de L. infantum en estos pacientes. Este hecho amplía el perfil de la epidemiología de la leishmaniosis tradicionalmente establecida en esta provincia. Los resultados muestran la conveniencia de usar la PCR como método de diagnóstico, debido a su alta sensibilidad y especificidad, así como a la posibilidad de tipificar la especie.

La detección temprana de la infección y de los casos asintomáticos mediante PCR podría ayudar a reducir la morbimortalidad de esta infección en el país.

\section{Agradecimientos}

Al personal médico que realizó el diagnóstico clínico, Gustavo Méndez, Sandra Borchichi y Liliana Arce; al Ministerio de Salud Pública de Misiones, por su ayuda para recabar los datos clínicos, y al Laboratorio de Alta Complejidad Misiones, por su aporte en este estudio.

\section{Referencias}

1. Organización Mundial de la Salud. Control of the leishmaniasis. Fecha de consulta: 19 de marzo de 2018. Disponible en: http://apps.who.int/iris/bitstream/10665/44412/1/WHO TRS 949 eng.pdf

2. Organización Mundial de la Salud. Investing to overcome the global impact of neglected tropical diseases. Third WHO report on neglected tropical diseases, 2015, WHO/HTM/ NTD/2015.1. Fecha de consulta: 19 de marzo 2018. Disponible en: http:apps.who.int/iris/bitstream/10665/152781/1/9789241564861 eng.pdf?ua=1

3. Dantas-Torres F. Canine leishmaniosis in South America. Parasit Vectors. 2009;26:S1. https://doi.org/10.1186/1756-3305-2-S1-S1

4. López K, Tartaglino LC, Steinhorst II, Santini MS, Salomón OD. Factores de riesgo, representaciones y prácticas asociadas con la leishmaniasis visceral humana en un foco urbano emergente en Posadas, Argentina. Biomédica. 2016;36:51-63. https://doi.org/10.7705/biomedica.v36i2.2953

5. Salomón OD, Mastrángelo AV, Santini MS, Liotta DJ, Yadón ZE. La eco-epidemiología retrospectiva como herramienta aplicada a la vigilancia de la leishmaniasis en Misiones, Argentina, 1920-2014. Rev Panam Salud Pública. 2016;40:29-39.

6. Alvar J, Yactayo S, Bern C. Leishmaniasis and poverty. Trends Parasitol. 2006;22:552-7. https://doi.org/10.1016/j.pt.2006.09.004

7. Alvar J, Cañavate C, Molina R, Moreno J, Nieto J. Canine leishmaniasis. Adv Parasitol. 2004;57:1-88. https://doi.org/10.1016/S0065-308X(04)57001-X 
8. Salomón OD, Sinagra A, Nevot MC, Barberian G, Paulin P, Estévez JO, et al. First visceral leishmaniasis focus in Argentina. Mem Inst Oswaldo Cruz. 2008;103:109-11. https://doi.org/10.1590/S0074-02762008000100018

9. Gutiérrez JA, Fleitas C, Milano M, González B. Informe epidemiológico semanal. Posadas: Ministerio de Salud Pública de Misiones; 2016.

10. Alvar J, Vélez ID, Bern C, Herrero M, Desjeux P, Cano J, et al. Leishmaniasis worldwide and global estimates of its incidence. PLoS One. 2012;7:e35671. https://doi.org/10.1371/journal.pone.0035671

11. Quintana MG, Fernández MS, Salomón OD. Distribution and abundance of Phlebotominae, vectors of leishmaniasis, in Argentina: spatial and temporal analysis at different scales. J Trop Med. 2012;2012:652803. https://doi.org/10.1155/2012/652803

12. Fernández MS, Santini MS, Cavia R, Sandoval AE, Pérez AA, Acardi S, et al. Spatial and temporal changes in Lutzomyia longipalpis abundance, a Leishmania infantum vector in an urban area in northeastern Argentina. Mem Inst Oswaldo Cruz. 2013;108: 817-24. https://doi.org/10.1590/0074-0276130047

13. Szelag EA, Parras MA, Fabiani M, Rosa JR, Salomón OD. Incipient colonisation of Lutzomyia longipalpis in the city of Resistencia, province of Chaco, Argentina (2010-2012). Mem Inst Oswaldo Cruz. 2014;109:488-91. https://doi.org/10.1590/0074-0276130054

14. Acosta L, Díaz R, Torres P, Silva G, Ramos M, Fattore G, et al. Identification of Leishmania infantum in Puerto Iguazú, Misiones, Argentina. Rev Inst Med Trop Sao Paulo. 2015;57:175-6. https://doi.org/10.1590/S0036-46652015000200013

15. Barroso PA, Nevot MC, Hoyos CL, Locatelli FM, Lauthier JJ, Ruybal P, et al. Genetic and clinical characterization of canine leishmaniasis caused by Leishmania (Leishmania) infantum in northeastern Argentina. Acta Tropica. 2015;150:218-23. https://doi.org/10.1016/j.actatropica.2015.08.007

16. Barroso PA, Marco JD, Locatelli FM, Cardozo RM, Mora MC, Hoyos CL, et al. Visceral leishmaniasis caused by Leishmania infantum in Salta, Argentina: Possible reservoirs and vectors. Am J Trop Med Hyg. 2015;93:334-9. https://doi.org/10.4269/ajtmh.14-0267

17. Gould IT, Perner MS, Santinin MS, Saavedra SB, Bezzi G, Maglianese MI, et al. Leishmaniasis visceral en la Argentina. Notificación y situación vectorial (2006-2012). Medicina (B. Aires). 2013;73:104-10.

18. Instituto Nacional de Estadísticas y Censos. Censo nacional del año 2010. Fecha de consulta: 14 de diciembre 2016. Disponible en: http://www.indec.gov.ar/nivel4 default. asp?id_tema_1=2\&id_tema_2=41\&id_tema_3=135

19. Schönian G, Nasereddin A, Dinse N, Schweynoch C, Schallig HD, Presber W, et al. PCR diagnosis and characterization of Leishmania in local and imported clinical samples. Diagn Microbiol Infect Dis. 2003;47:349-58. https://doi.org/10.1016/S0732-8893(03)00093-2

20. Cruz I, Millet A, Carrillo E, Chenik M, Salotra P, Verma S, et al. An approach for interlaboratory comparison of conventional and real-time PCR assays for diagnosis of human leishmaniasis. Exp Parasitol. 2013;134:281-9. https://doi.org/10.1016/j.exppara.2013.03.026

21. Salomón OD, Sosa SE, Rossi GC, Spinelli G. Presencia de Lutzomyia longipalpis y situación de la leishmaniasis visceral en Argentina. Medicina (B. Aires). 2001;61:174-8.

22. Martín-Sánchez J, Navarro-Mari JM, Pasquau-Liaño J, Salomón OD, Morillas-Márquez F. Visceral leishmaniasis caused by Leishmania infantum in a Spanish patient in Argentina: What is the origin of the infection? Case Report. BMC Infect Dis. 2004;4:20. https://doi.org/10.1186/1471-2334-4-20

23. Salomón OD, Sosa SE, Canini L, Córdoba EL. Leishmaniosis tegumentaria en un área con niveles epidémicos de transmisión, Salta, Argentina, 1998. Medicina (B. Aires). 2001;61:284-90.

24. Ministerio de Salud de la Nación. Leishmaniasis visceral. Fecha de consulta: 14 de diciembre 2016. Disponible en: http://www.msal.gob.ar/images/stories/bes/graficos/0000000 798cnt-2012-03-15_leishmaniasis-visceral-guia.pdf

25. Guerin PJ, Olliaro P, Sundar S, Boelaert M, Croft SL, Desjeux P, et al. Visceral leishmaniasis: Current status of control, diagnosis, and treatment, and a proposed research and development agenda. Lancet Infect Dis. 2002;2:494-501. https://doi.org/10.1016/S1473-3099(02)00347-X

26. Cortes S, Chicharro C, Cruz I, Cristovao JM, Canavate C, Campino L. Genetic diversity of human zoonotic leishmaniasis in Iberian Peninsula. Zoo Pub Heal. 2010;58:234-7. https://doi.org/10.1111/j.1863-2378.2010.01374.x 
27. Solano-Gallego L, Koutinas A, Miró G, Cardoso L, Pennisi MG, Ferrer L, et al. Directions for the diagnosis, clinical staging, treatment and prevention of canine leishmaniasis. Vet Parasitol. 2009;165:1-18. https://doi.org/10.1016/j.vetpar.2009.05.022

28. Fraga LT, Brustoloni YM, Lima RB, Cavalheiros MB, Oshiro ET, Oliveira J, et al. Polymerase chain reaction of peripheral blood as a tool for the diagnosis of visceral leishmaniasis in children. Mem Inst Oswaldo Cruz. 2010;105:310-3. https://doi.org/10.1590/0037-8682-0145-2013

29. Antinori S, Calattini S, Longhi E, Bestetti G, Piolini R, Magni C, et al. Clinical use of polymerase chain reaction performed on peripheral blood and bone marrow samples for the diagnosis and monitoring of visceral leishmaniasis in HIV-infected and HIV uninfected patients: A single-center, 8-year experience in Italy and review of the literature. Clin Infect Dis. 2007;44:1602-10. https://doi.org/10.1086/518167

30. Cortés S, Rolao N, Ramada J, Campino L. PCR as a rapid and sensitive tool in the diagnosis of human and canine leishmaniasis using Leishmania donovani s.l.-specific kinetoplastid primers. Trans Roy Soc Trop Med Hyg. 2004;98:12-7. https://doi.org/10.1016/S0035-9203(03)00002-6

31. Roelfsema JH, Nozari N, Herremans T, Kortbeek LM, Pinelli E. Evaluation and improvement of two PCR targets in molecular typing of clinical samples of Leishmania patients. Exp Parasitol. 2010;10:1006. https://doi.org/10.1016/j.exppara.2010.06.024

32. Bruhn FR, Morais HF, Bruhn NC, Cardoso DL. Human visceral leishmaniasis: Factors associated with deaths in Belo Horizonte, Minas Gerais state, Brazil from 2006 to 2013. Epidemiol Infect. 2018;146:565-70. https://doi.org/10.1017/S0950268818000109

33. Lima ID, Lima AL, Mendes-Aguiar CO, Coutinho JF, Wilson ME, Pearson RD, et al. Changing demographics of visceral leishmaniasis in northeast Brazil: Lessons for the future. PLoS Negl Trop Dis. 2018;12:e0006164. https://doi.org/10.1371/journal.pntd.0006164

34. Peres D, Lamounier D, López I, Nery C, Martins P. Short report: Heterogeneity of Leishmania infantum chagasi kinetoplast DNA in Teresita (Brazil). Am Soc Trop Med Hyg. 2010;82:819-21. https://doi.org/10.4269/ajtmh.2010.09-0600

35. Gómez AE. Análisis de la diversidad genética de Leishmania infantum en Paraguay mediante la técnica del kDNA-PCR-RFLP (tesis). Asunción: Universidad Nacional de Asunción; 2013.

36. Figueiró-Filho EA, Duarte G, El-Beitune P, Quintana SM, Lemos Maia T. Visceral leishmaniasis (kala-azar) and pregnancy. Infect Dis Obstet Gynecol. 2004;12:31-40. https://doi.org/10.1080/1064744042000210384

37. Mescouto-Borges MM, Maués E, Costa DL, da Silva Pranchevicius MC, Sierra Romero GA. Congenitally transmitted visceral leishmaniasis: Report of two Brazilian human cases. Braz J Infect Dis. 2013;17:263-6. https://doi.org/10.1016/j.bjid.2012.10.017 